\title{
STOTE \\ Surgical treatment of an abnormally positioned right adrenal tumor on segmental caudal vena cava aplasia in two dogs
}

\author{
Satoshi TAKAGI ${ }^{1,2) *}$, Eiichi KANAI ${ }^{1)}$, Keitaro MORISHITA ${ }^{2)}$, Hiroko OGAWA ${ }^{3)}$ and \\ Junya OGAWA ${ }^{3)}$ \\ 1)Laboratory of Small Animal Surgery, Department of Veterinary Medicine, Azabu University, \\ 1-17-71 Fuchinobe, Chuo-ku, Sagamihara, Kanagawa 252-5201, Japan \\ 2) Veterinary Teaching Hospital, Graduate School of Veterinary Medicine, Hokkaido University, \\ Kita-ku N18 W9, Sapporo, Hokkaido 060-0818, Japan \\ 3) Ogawa Canine Feline Hospital, 1-9-14 Tsunishi, Kamakura 248-0034, Japan
}

J. Vet. Med. Sci.

83(1): 69-72, 2021

doi: 10.1292/jvms.20-0584

Received: 4 October 2020

Accepted: 2 November 2020

Advanced Epub:

16 November 2020
ABSTRACT. Segmental caudal vena cava (CVC) aplasia is a rare congenital vascular anomaly in dogs. Two dogs were diagnosed by CT imaging to have right adrenal tumors with concomitant segmental CVC aplasia. During surgery, a firm connection between the right adrenal gland and CVC was observed in both cases. The adrenal glands were found ventral to the CVC and the adrenal tumor was resected including the vascular wall. CVC venectomy for tumor removal will be required if the right adrenal gland is displaced in dogs with segmental CVC aplasia, even if there is no intravascular invasion.

KEY WORDS: adrenal tumor, dog, segmental caudal vena cava aplasia

Segmental caudal vena cava (CVC) aplasia is a congenital vascular anomaly with sporadic incidence in dogs. This malformation is usually observed incidentally on diagnostic abdominal imaging or autopsy since most cases are asymptomatic [1, 2, 9]. In dogs, vascular invasion is relatively common with adrenal tumors. Among them, pheochromocytomas are more likely to develop tumor thrombus into the CVC via the phrenicoabdominal vein [7]. Several surgical procedures have been described for CVC, including portosystemic shunts (PSS) and thrombi formation [2,9]. However, the influence of an anatomical abnormality in determining the choice of surgical technique for segmental CVC is not well known. Herein, we describe the diagnosis and successful surgical management of segmental CVC in two dogs with right-sided adrenal tumors.

Case 1: A 10-year-old castrated male, Papillon, weighing $8.7 \mathrm{~kg}$ showed elevated alkaline phosphatase ( $>3,500 \mathrm{IU} / 1$, reference range: 47-254 IU/1) in a comprehensive medical examination. A referral veterinarian performed an abdominal ultrasound examination to inspect the cause of this abnormality, and an enlarged right adrenal gland (14-mm) was found. Furthermore, the size and location of the right adrenal gland were also abnormal. Initially, the right adrenal gland did not seem to be located dorsal to the caudal vena cava (CVC); however, the precise position could not be identified due to the restless nature of the dog. The dog underwent an adrenocorticotropic hormone $(\mathrm{ACTH})$ stimulation test, and the results were within normal limits. Adrenalectomy was scheduled, and preoperative CT imaging was performed for surgical planning. The CT scan showed that the tumor was located ventral to the CVC, but abnormal vessel anatomy was not observed, and dog was diagnosed with segmental CVC aplasia immediately before surgery. There was no vascular invasion observed into the CVC (Fig. 1).

Intraoperatively, a firm connection was found between the right adrenal gland and CVC. Transient vascular occlusion was performed using Rummel tourniquets during the tumor resection. A portion of the vascular wall of the CVC was removed, followed by reconstruction of the CVC wall defect by continuous suture pattern using 5-0 polypropylene suture (PROLENE: Johnson and Johnson, New Brunswick, NJ, USA). During anesthesia monitoring during the surgery, no cardiovascular abnormalities were observed. The dog recovered uneventfully. An anticoagulant (heparin sulphate, 75 units $/ \mathrm{kg}$, SC: subcutaneously, tid) was administered before surgery and was maintained until the next morning. The tumor was histopathologically diagnosed as a concurrent adrenocortical adenocarcinoma and pheochromocytoma. The dog expired 1,005 days postoperatively due to an unrelated cause.

Case 2: An 11-year-old intact female, Miniature Schnauzer, weighing $11.6 \mathrm{~kg}$, was diagnosed with a 13-mm non-functional right adrenal tumor during an ultrasonographic assessment for diarrhea and vaginal discharge that persisted for a week. The position of 

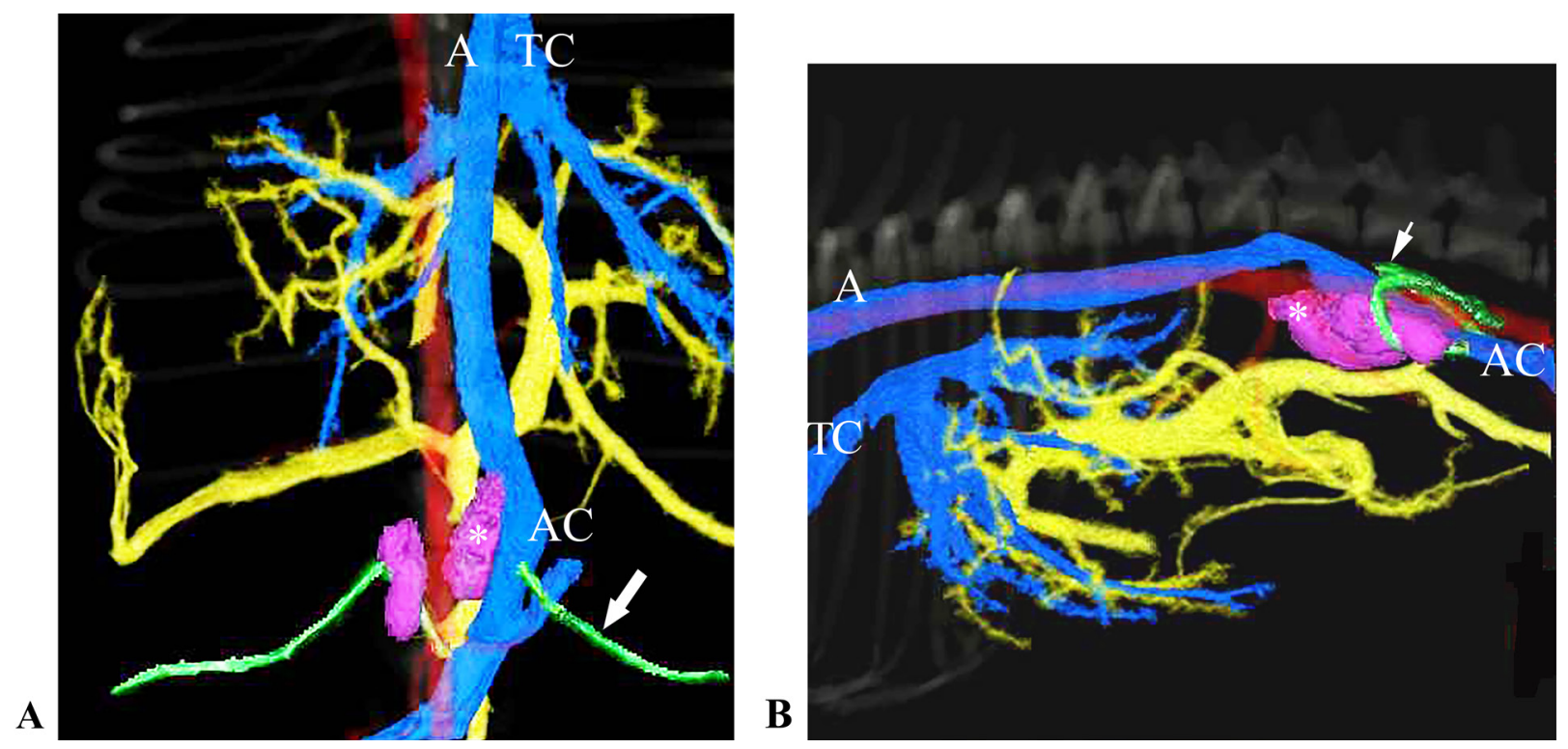

Fig. 1. A) Dorsal view of a reconstructed 3D abdominal CT image in case 1 . The left side of the figure is the left side of the dog. B) Right lateral recumbency view of a reconstructed 3D abdominal CT image. A direct shunting from the caudal vena cava (CVC) to the azygous vein was identified, and this case was diagnosed as a right-lateral cavo-right-azygous shunt. The enlarged right adrenal gland $\left({ }^{*}\right)$ existed ventrally to the CVC, while the common trunk (formerly named as phrenicoabdominal vein: white arrow) was connected at the dorsal part of the CVC. A: Azygous vein, TC: thoracic portion of the CVC, AC: abdominal portion of the CVC.

the right adrenal gland was ventral to the CVC (Fig. 2). Preoperative abdominal CT imaging was performed for surgical planning. The dog was diagnosed to have an adrenal tumor with concomitant segmental CVC aplasia (Fig. 3) and pyometra. Similar to the first case, venous invasion of the adrenal tumor was not observed. During surgery, the right adrenal tumor was bluntly dissected. It was observed to be firmly attached to the CVC (Fig. 4). The tumor was removed with transient occlusion of the CVC using Satinsky forceps. Vascular wall resection and reconstruction were performed using the same procedures as in case 1 . The dog recovered uneventfully. An anticoagulant (heparin sulphate, 75 units $/ \mathrm{kg}, \mathrm{SC}$, tid) was administered before surgery and was maintained until the next morning. The histopathological diagnosis was adrenocortical adenocarcinoma. This dog died 737 days postoperatively due to an unrelated cause.

Segmental CVC aplasia, also known as azygos continuation of the CVC or CVC uniting with the azygos vein, is very rare congenital vascular anomaly in dogs $[1,2]$. The incidence of CVC without other anomalies is approximately $1-3 \%$ in dogs. This is usually observed on diagnostic abdominal imaging or on autopsy [1,2]. Segmental CVC aplasia is rare in dogs, but more cases are being diagnosed due to the increase in use of CT scan for abdominal diseases [2]. In fact, in the first case under consideration, segmental CVC was overlooked on preoperative CT imaging. Since most cases are asymptomatic, the true incidence of segmental CVC could be much higher than its reported incidence.

There are seven types of CVC aplasia, classified by Schwartz et al. as follows: type 1, right lateral cavo-right-azygos shunt; type 2, right medial cavo-right-azygos shunt and small blind ending CVC cranial to the left kidney; type 3, large aneurysmal right medial cavo-right-azygos shunt with an isthmian connection to the azygos vein; type 4, split CVC and right medioventral cavoright-azygos shunt; type 5, dorsal cavo-right-azygos shunt; type 6, aneurysmal cavo-left-azygos shunt with connecting completely shunting portal vein; and type 7, split CVC, aneurysmal cavo-left-azygos shunt, and connecting portal vein shunt vessel [9]. Nine of the 37 reported cases had concurrent PSS [9]. In this case report, CT examination was conducted for the two dogs who developed right adrenal tumor with segmental CVC aplasia and the cases were identified as right-lateral cavo-right-azygos shunts (Type 1) [9]. The two cases were thoroughly assessed for the presence of shunt vessels and no vascular abnormalities were seen other than segmental CVC aplasia.

Most cases are asymptomatic and are usually identified as an incidental finding as the cases in this report [9]. Besides the concurrent PSS, this vascular abnormality poses certain risks, such as thrombosis [3, 5, 9]. Among the three reported cases, one dog was successfully managed with multiple surgeries and administration of aspirin [3], one dog died intraoperatively due to poor condition [5], and in one dog, the large thrombi were left without treatment and it survived for more than 1.5 years with no clinical signs [5].

Surgery is uncommon in dogs with segmental CVC aplasia because most cases are asymptomatic [9]. It is not known whether the anatomical differences from normal dogs could affect the treatment of surgical diseases. However, it was expected to have some difficulty in surgical intervention for abdominal disease, especially in the area between the right kidney and the liver in right lateral cavo-right-azygos shunt cases. In fact, the two cases in this report suggested that this abnormality could make removal of the right adrenal gland difficult. 


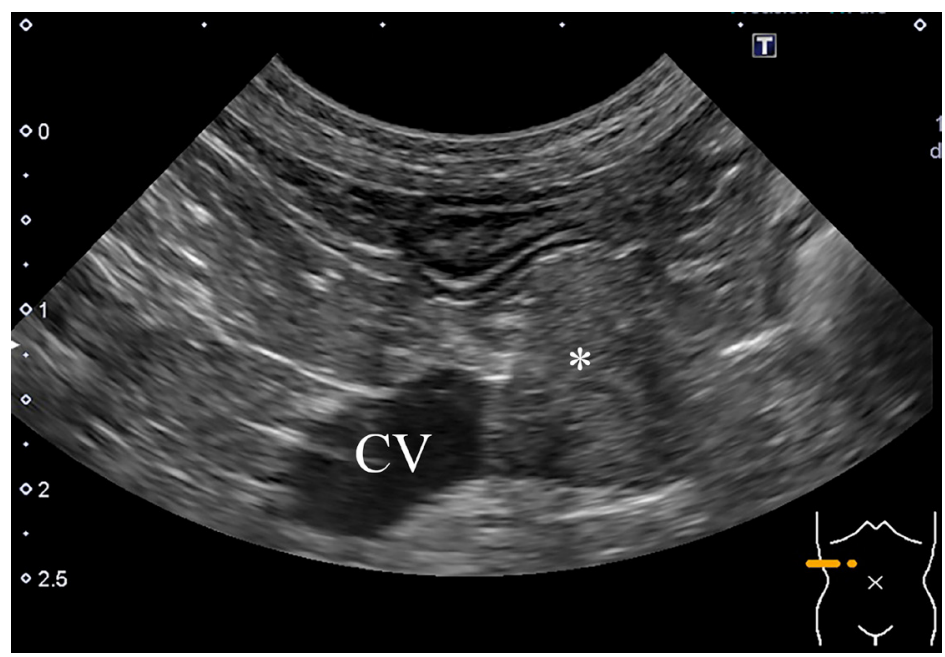

Fig. 2. Short axis image of the right adrenal gland obtained by abdominal ultrasound in case 2 . The neoplastic right adrenal gland (*) was displaced ventrally to the caudal vena cava (CVC). The left side of the figure is the right side of the dog, and this view also shows the medially located adrenal gland compared to the normal position. CV: caudal vena cava.

A
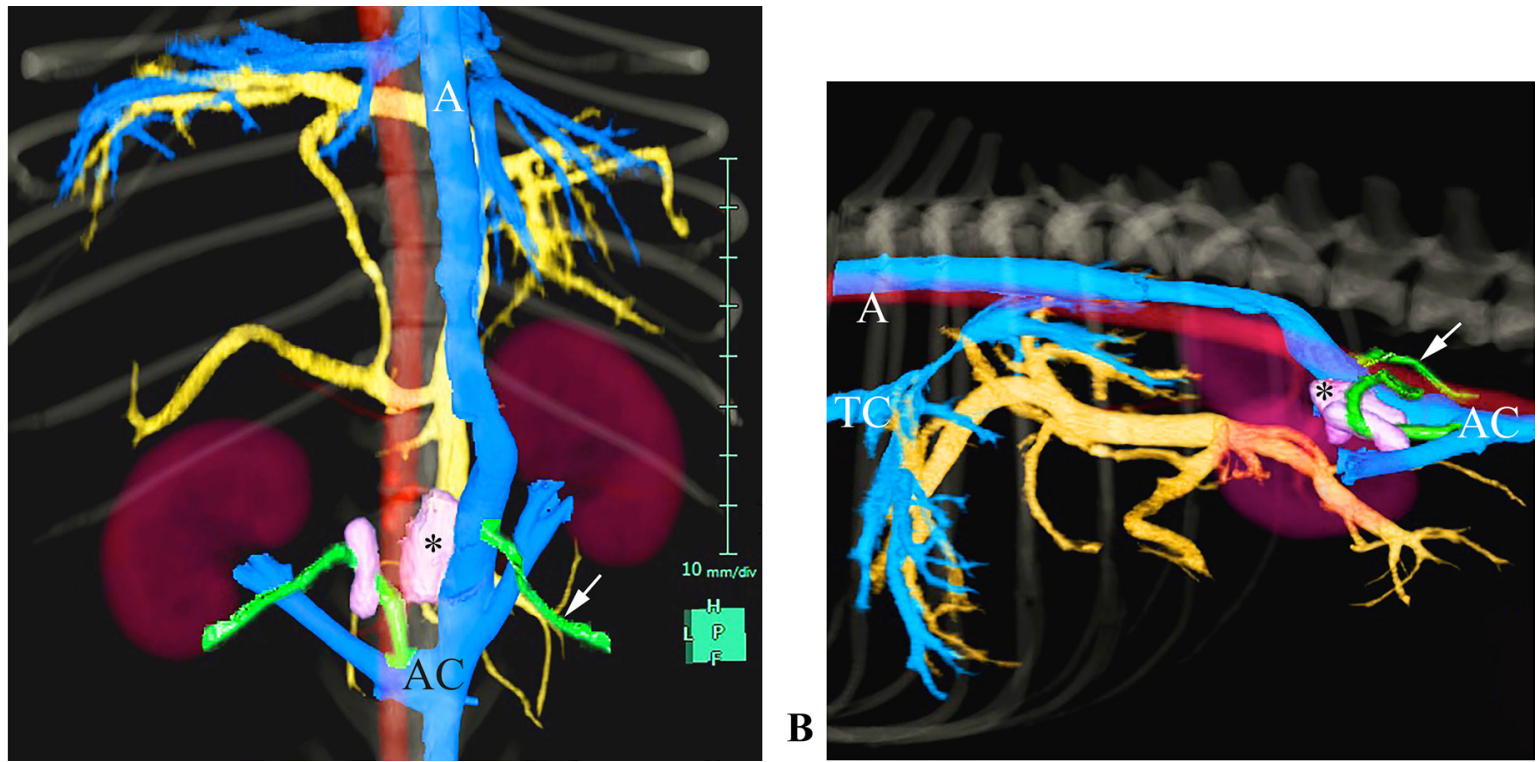

Fig. 3. A) Dorsal view of a reconstructed 3D abdominal CT image in case 2. The left side of the figure is left of the dog. B) Right lateral recumbency view of a reconstructed 3D abdominal CT image. A direct shunting from the caudal vena cava (CVC) to the azygous vein was identified, and this case was also diagnosed as a right-lateral cavo-right-azygous shunt. The enlarged right adrenal gland (*) existed ventrally to the $\mathrm{CVC}$, while the common trunk (formerly named as phrenicoabdominal vein: white arrow) was connected at the dorsal part of the CVC. All these findings are the same as in case 1. A: Azygous vein, TC: thoracic portion of the CVC, AC: abdominal portion of the CVC.

In normal dogs, the outflow of blood from the adrenal gland into the CVC is through the adrenal vein and then returns through the common trunk (formerly known as the phrenicoabdominal vein) [4]. Adrenal tumors often develop vascular invasion into the CVC via the common trunk $[6,7]$. In these cases, vessel occlusion is required and is performed using Rummel tourniquets and Satinsky forceps $[6,7]$. In our cases, the normal right common trunks were present, which branched and coursed in the normal position, and in the opposite side of the displaced right adrenal gland.

The prehepatic CVC is from the embryologic subcardinal veins, and the renal part of the CVC originates from the subcardinal and supracardinal veins $[5,8]$. In the present cases, the subcardinal veins may have degenerated while a remnant of the supracardinal vein influenced the abnormal running of the right common trunk. It was strongly suggested that due to this abnormality in the vessel anatomy, the right adrenal glands were directly attached to the CVC. Because of this finding, further dissection can cause severe bleeding from a punctured CVC during surgical manipulation. Besides, considering the principle of 


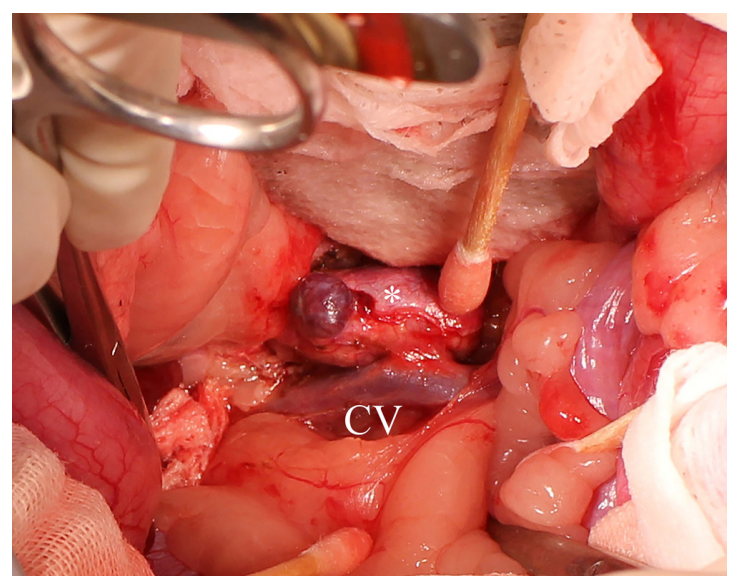

Fig. 4. Intraoperative view of case 2 for adrenalectomy. The surrounding tissue was dissected. The left side of the figure shows the cranial side of the dog. A neoplastic adrenal gland (*) was firmly attached to the caudal vena cava (CVC) without vascular invasion. CV: CVC.

surgical oncology, the CVC vessel wall was considered as the tumor margin. Therefore, they should be resected with adequate vascular occlusion techniques even if they have no vascular invasion. With these considerations, it is important to evaluate the anatomy of the $\mathrm{CVC}$ first before resecting the right adrenal tumor. Additionally, this technique requires relatively advanced surgical skills, and in the present cases, surgery was performed by the same board-certified surgeon (Takagi: Diplomate of the Japanese college of veterinary surgeons).

In normal dogs, the right adrenal gland is located dorsal to the CVC [4], but in our experience, the abnormal location of the right adrenal gland relative to the CVC suggests a congenital abnormality. The right common trunk receives the right caudal phrenic vein and the right cranial abdominal vein [4], and these vessels connect to the diaphragm and dorsal muscles, which contribute to the dorsally fixed right adrenal gland. In the present report, the adrenal glands were displaced ventrally to the CVC. Furthermore, the right adrenal gland was medially displaced, as confirmed by CT images. The ventral displacement of the right adrenal gland by abdominal ultrasound may predict the existence of type 1 segmental CVC aplasia.

In conclusion, if a right adrenal gland tumor is found to be displaced in dogs with concomitant segmental CVC aplasia, CVC venectomy is required, even if there is no intravascular invasion.

POTENTIAL CONFLICTS OF INTEREST. The authors have nothing to disclose.

ACKNOWLEDGMENT. The author would like to thank Mr. Yoshihisa Yamane (Hokkaido University Veterinary Teaching Hospital) for his help in making 3D CT images.

\section{REFERENCES}

1. Buchanan, J. W. 1992. Causes and prevalence of cardiovascular disease. pp. 647-652. In: Current Veterinary Therapy XI (Kirk, R. W. and Bonagura, J. D. eds.), Saunders, Philadelphia.

2. Fischetti, A. J. and Kovak, J. 2008. Imaging diagnosis: azygous continuation of the caudal vena cava with and without portocaval shunting. Vet. Radiol. Ultrasound 49: 573-576. [Medline] [CrossRef]

3. Harder, M. A., Fowler, D., Pharr, J. W., Tryon, K. A. and Shmon, C. 2002. Segmental aplasia of the caudal vena cava in a dog. Can. Vet. J. 43: 365-368. [Medline]

4. Hermanson, J. W., de Lahunta, A. and Evans, H. E. 2019. The veins. pp. 595-597. In: Miller's Anatomy of the Dog, 5th ed. (Hermanson, J. W., de Lahunta, A. and Evans, H. E. eds.), Elsevier, St. Louis.

5. Hunt, G. B., Bellenger, C. R., Borg, R., Youmans, K. R., Tisdall, P. L. and Malik, R. 1998. Congenital interruption of the portal vein and caudal vena cava in dogs: six case reports and a review of the literature. Vet. Surg. 27: 203-215. [Medline] [CrossRef]

6. Knight, R. C., Lamb, C. R., Brockman, D. J. and Lipscomb, V. J. 2019. Variations in surgical technique for adrenalectomy with caudal vena cava venotomy in 19 dogs. Vet. Surg. 48: 751-759. [Medline] [CrossRef]

7. Mayhew, P. D., Culp, W. T. N., Balsa, I. M. and Zwingenberger, A. L. 2018. Phrenicoabdominal venotomy for tumor thrombectomy in dogs with adrenal neoplasia and suspected vena caval invasion. Vet. Surg. 47: 227-235. [Medline] [CrossRef]

8. McGeady, T. A., Quinn, P. J., FitzPatrick, E. S., Ryan, M. T., Kilroy, D. and Lonergan, P. 2017. Cardiovascular system. pp. 119-147. In: Veterinary Embryology, 2nd ed. (McGeady, T. A., Quinn, P. J., FitzPatrick, E. S., Ryan, M. T., Kilroy, D., Lonergan, P. eds.), John Wiley \& Sons, Chichester.

9. Schwarz, T., Rossi, F., Wray, J. D., Ablad, B., Beal, M. W., Kinns, J., Seiler, G. S., Dennis, R., McConnell, J. F. and Costello, M. 2009. Computed tomographic and magnetic resonance imaging features of canine segmental caudal vena cava aplasia. J. Small Anim. Pract. 50: 341-349. [Medline] [CrossRef] 